Article

\title{
Human Movement Detection and Identification Using Pyroelectric Infrared Sensors
}

\author{
Jaeseok Yun* and Sang-Shin Lee
}

Embedded Software Convergence Research Center, Korea Electronics Technology Institute, 25 Saenari-ro, Bundang-gu, Seongnam 463070, Korea; E-Mail: sslee@keti.re.kr

* Author to whom correspondence should be addressed; E-Mail: jaeseok@ keti.re.kr; Tel.: +82-31-789-7581; Fax: +82-31-789-7589.

Received: 26 February 2014; in revised form: 11 April 2014 / Accepted: 24 April 2014 / Published: 5 May 2014

\begin{abstract}
Pyroelectric infrared (PIR) sensors are widely used as a presence trigger, but the analog output of PIR sensors depends on several other aspects, including the distance of the body from the PIR sensor, the direction and speed of movement, the body shape and gait. In this paper, we present an empirical study of human movement detection and identification using a set of PIR sensors. We have developed a data collection module having two pairs of PIR sensors orthogonally aligned and modified Fresnel lenses. We have placed three PIR-based modules in a hallway for monitoring people; one module on the ceiling; two modules on opposite walls facing each other. We have collected a data set from eight subjects when walking in three different conditions: two directions (back and forth), three distance intervals (close to one wall sensor, in the middle, close to the other wall sensor) and three speed levels (slow, moderate, fast). We have used two types of feature sets: a raw data set and a reduced feature set composed of amplitude and time to peaks; and passage duration extracted from each PIR sensor. We have performed classification analysis with well-known machine learning algorithms, including instance-based learning and support vector machine. Our findings show that with the raw data set captured from a single PIR sensor of each of the three modules, we could achieve more than $92 \%$ accuracy in classifying the direction and speed of movement, the distance interval and identifying subjects. We could also achieve more than $94 \%$ accuracy in classifying the direction, speed and distance and identifying subjects using the reduced feature set extracted from two pairs of PIR sensors of each of the three modules.
\end{abstract}


Keywords: pyroelectric infrared sensor; human movement detection; human identification; machine learning; occupancy sensing; occupant localization

\section{Introduction}

With the advancement of sensor and actuator technologies, our indoor environment, such as buildings, has been instrumented with various sensors, including temperature, humidity, illumination, $\mathrm{CO}_{2}$ and occupancy sensor, and, thus, can be aware of changes in the user's state and surrounding, finally controlling building utilities to adapt their services and resources to the user's context, e.g., automatic lighting control, heating, ventilation and air-conditioning (HVAC) system adjustment, electrical outlet turn-off, unusual behavior detection and home invasion prevention. Such context-aware systems have deployed occupant location as the principal form of the user's context. Accordingly, indoor tracking and localization is one of the key technologies for providing activity-aware services in a smart environment.

Pyroelectric infrared (PIR) sensors are well-known occupancy detectors. They have been widely employed for human tracking systems, due to their low cost and power consumption, small form factor and unobtrusive and privacy-preserving interaction. In particular, a dense array of PIR sensors having digital output and the modulated visibility of Fresnel lenses can provide capabilities for tracking human motion, identifying walking subject and counting people entering or leaving the entrance of a room or building. However, the analog output signal of PIR sensors involves more aspects beyond simple people presence, including the distance of the body from the PIR sensor, the velocity of the movement (i.e., direction and speed), body shape and gait (i.e., a particular way or manner of walking). Thus, we can leverage discriminative features of the analog output signal of PIR sensors in order to develop various applications for indoor human tracking and localization.

In this paper, we present an empirical study of human movement detection and identification using PIR-based modules having two pairs of orthogonally-aligned PIR sensors. We have developed a data collection module consisting of two pairs of PIR sensors whose dual sensing elements are orthogonally aligned and Fresnel lenses are modified to narrow the field of view of the PIR sensors to its horizontal motion plane, a data logger, op-amp circuits and a rechargeable battery. We have placed three PIR-based modules in a hallway for monitoring people; one PIR-based module is placed on the ceiling; two PIR-based modules are placed on opposite walls facing each other. We have collected a data set from eight experimental subjects when walking in three different conditions: two directions (back and forth), three distance intervals (close to one wall sensor, in the middle, close to the other wall sensor) and three speed levels (slow, moderate, fast). We have employed two types of feature sets: a raw data set and reduced feature set composed of amplitude and time to peaks; and passage duration extracted from each PIR sensor. We have performed classification analysis according to various configurations, including the number of modules involved (ceiling-mounted module $v s$. wall-mounted modules), the number of PIR sensors involved (a single PIR sensor, a pair of PIR sensors orthogonally aligned and two pairs of PIR sensors orthogonally aligned), the feature set (raw data set $v s$. reduced feature set) and well-known machine learning algorithms, including instance-based learning and support vector machine. Our findings show that with the raw data set captured from a single PIR sensor of each of 
the three modules, we were able to achieve more than $92 \%$ correct detection of direction and speed of movement, the distance interval and identification of walking subjects. We could also achieve more than $94 \%$ accuracy in classifying the direction, speed level and distance interval and identifying walking subjects using the reduced feature set extracted from each of the three modules equipped with two pairs of PIR sensors.

The rest of the paper is organized as follows: Section 2 introduces various indoor localization and tracking and motion detecting systems using PIR sensors. Section 3 presents a human movement detection and identification system and explains what aspects of PIR sensors we employ to detect the direction and speed of movement and the distance interval. Section 4 describes the PIR sensor-based movement detecting device and data collection procedure and explains which features we extract from the raw data set and which classifiers we employ for machine learning. Section 5 presents the experimental results of the classification analysis with the raw data set and reduced feature set extracted from the raw data set collected. Section 6 discusses remaining challenges for our methods. Finally, Section 7 offers concluding remarks.

\section{Related Work}

\subsection{PIR Sensor-Based Applications in Smart Environments}

PIR sensors are commonly used with a variety of sensors in diverse applications for building smart environments, such as healthcare, smart energy system and security. Han et al. presented an occupancy and indoor environment quality sensing method based on a suite of sensors, including PIR sensors, $\mathrm{CO}_{2}$ sensors, humidity sensors and concentration sensors [1]. Tsai et al. illustrated a way of reducing the standby power consumption of lighting devices based on a PIR sensor, an ambient light sensor and lighting duration modules [2]. They also demonstrated a way of reducing the standby power consumption of a personal computer monitor in sleep status [3]. Erickson et al. implemented a power-efficient occupancy-based energy management system based on camera-based and PIR sensor-based wireless sensor networks for opportunistically controlling HVAC system and, thus, increasing energy efficiency, while maintaining conditioning effectiveness [4]. This capability of PIR sensor-based occupancy and motion detection for diverse application domains provided the motivation for this research into the feasibility of a human movement detection system using various features extracted from the PIR sensor signal.

\subsection{Indoor Human Tracking with PIR Sensors}

Many researchers have devoted a great deal of effort to developing localization technology for indoor human tracking using PIR sensors. Gopinathan et al. developed a pyroelectric motion tracking system based on coded apertures, which could detect human motion in one of the 15 cells in a 1.6-m square area using four PIR detectors [5]. Shankar et al. developed a human tracking system using a low-cost sensor cluster consisting of PIR sensors and Fresnel lens arrays to implement the desired spatial segmentations [6]. They analyzed the response characteristics of the sensor cluster and extracted the velocity and the direction of motion over large areas of over $12 \mathrm{~m}$. Hao et al. presented a human 
tracking system using an MSP430 family microcontroller, an RF transceiver and a radial sensor module with eight PIR detectors with Fresnel lens arrays arranged around a circle [7]. They showed that the system can be used to track a single human target by detecting its angular displacement while moving. Luo et al. demonstrated a 3D simulation study for human tracking using PIR sensors [8]. Their approach showed the visibility modulation of each sensor detector and the layout of sensor modules and proposed algorithms for localizing and tracking people based on the binary output of the PIR sensors.

\subsection{Human Movement Detection with PIR Digital Outputs}

More specifically, researchers have been working on detecting movement direction and counting people entering or leaving the entrance of a room or building using the on-off output signal of PIR sensors. Hashimoto et al. presented a people counting system composed of a one-dimensional eight-element custom-fabricated array detector, an IR-transparent lens and an oscillating mechanical chopper [9]. They show a 99\% recognition accuracy of the two-way back-and-forth moving direction and a 95\% recognition accuracy of the number of passersby. Wahl et al. developed a people counting system for an office space based on self-sustaining ultra-low power sensor nodes composed of a pair of uni-directional PIR sensors [10]. They simply differentiate the direction of movement at a gateway by observing the time difference between inward-facing and outward-facing PIR sensors. Through a real-life office room study, they show their prototype system performs well on detecting all occupant movements to and from the office room with a very low error rate $(<1 \%)$. They also proposed an approach to estimate people count per office space using distributed pairs of PIR sensors and an algorithm to process the distributed sensor information [11].

\subsection{Human Movement Detection with PIR Analog Outputs}

Lee proposed a novel method of detecting the motion direction for an object moving in the field of view of a single PIR sensor, whose dual sensing elements are reversely polarized and aligned in the motion plane of the PIR sensor [12]. As Lee presented, the analog output signal of PIR sensors involves more aspects beyond simple on-off triggering, and such features have been exploited in several ways for recognizing motion direction. Zappi et al. built a low-cost PIR sensor-based wireless network system for detecting the direction of movement and distinguishing the number of people (two and three) walking in line, as well as walking side by side in a hallway [13]. They showed the $100 \%$ correct detection of direction of movement and $89 \%$ correct detection of the number of people. The authors also built a cluster system composed of two PIR sensors facing each other in a hallway for detecting the direction of movement and distance intervals (close to one sensor, in the middle, close to the other sensor) when a person is walking $[14,15]$. They extracted the passage duration and output amplitude from the PIR output signals and performed classification analysis using the support vector machine and $k$-nearest neighbor algorithms. The experimental results show $100 \%$ correct detection of the direction of movement and $83.49 \%-95.35 \%$ correct detection of distance intervals. More recently, Yun and Song presented a novel method of detecting the relative direction of human movement in eight directions uniformly distributed with two pairs of PIR sensors whose sensing elements are orthogonally aligned [16]. With the raw data sets captured from two orthogonally-aligned PIR sensors with modified lenses, they achieved more than 
a $98 \%$ correct detection of the direction of movement. They also found that with the reduced feature set composed of three peak values in the time domain for each PIR sensor, they were able to achieve $89 \%-95 \%$ recognition accuracy according to machine learning algorithms.

\subsection{Human Identification with PIR Sensors}

Many researchers have also been working on a human identification system using PIR sensors. Fang et al. presented a human identification system using a PIR sensor whose visibility is modulated by a Fresnel lens array and a principal components regression method [17]. They investigated the performance of the identification system in terms of the optimal element number of the lens array, the height of the sensor location, the sensor-to-object distance and walking speed. Fang et al. presented a method for identifying subjects walking through a dependent and an independent path using PIR sensors with modulated visibilities and hidden Markov models [18]. The average recognition rates for 10 persons are $91 \%$ and $78.5 \%$ in the path-dependent and path-independent cases, respectively. They also demonstrated a biometrics system for open-set walker recognition using a PIR sensor, a Fresnel lens array and the maximum likelihood principal components estimation (MLPCE) method [19]. Hao et al. introduced a wireless distributed PIR system for tracking and identifying multiple humans based on their body heat radiation and gait [20]. For a single walker identification, the experiment for five persons with sensor fusion and four PIR modules, each of which is composed of eight PIR sensors, shows 100\% recognition accuracy. Tao et al. presented a person localization algorithm using an infrared ceiling sensor network for providing various personalized services in an office environment [21]. The result shows $84 \%$ accuracy on recognizing five persons with support vector machine. Recently, Sun et al. presented a multi-target, complex scenario identification system based on a binary PIR-based sensor network [22].

However, the previous research for human identification exploited the digital on-off output signals of PIR sensors with visibility modulation, and it is worth investigating the availability of analog output signals of PIR sensors for identifying subjects, as in the research on classifying the walking direction and speed and the distance of the body from the sensor. To our knowledge, there is no published literature investigating human identification based on the analog output signals of PIR sensors and machine learning algorithms. We also consider various sensor configurations, such as the number of sensors involved and different sensor placement, including ceiling-mounted and wall-mounted ones, to achieve high recognition accuracy in human movement detection and identification. In addition, we perform an extensive study using a larger number of classification algorithms than previous ones, helping people choose appropriate algorithms for their PIR-based applications.

\section{Detecting Human Movement and Sensors}

\subsection{Human Movement Detection and Identification}

In order to build a smart environment where systems could understand the activities a user is involved with and the surroundings and then adapting their services and resources to the user's context, we need to develop a robust motion detection and tracking system using various sensors. Many researchers have 
devoted their efforts toward building robust motion tracking systems using vision-based sensors, i.e., cameras. The research projects based on vision-based sensors mainly consider position, speed, direction, shape and size (i.e., the number of pixels in cameras) as the principal context for identifying the users and understanding their activities [23]. Accordingly, as shown in Figure 1, a motion tracking system will need to robustly detect: (1) the identity of the moving object; (2) at which location the object is; (3) to which direction the object is moving; and (4) how fast the object is moving. We next consider the sensing systems for human movement detection and identification for building a robust motion tracking system.

Figure 1. Human movement detection and identification for indoor person tracking.

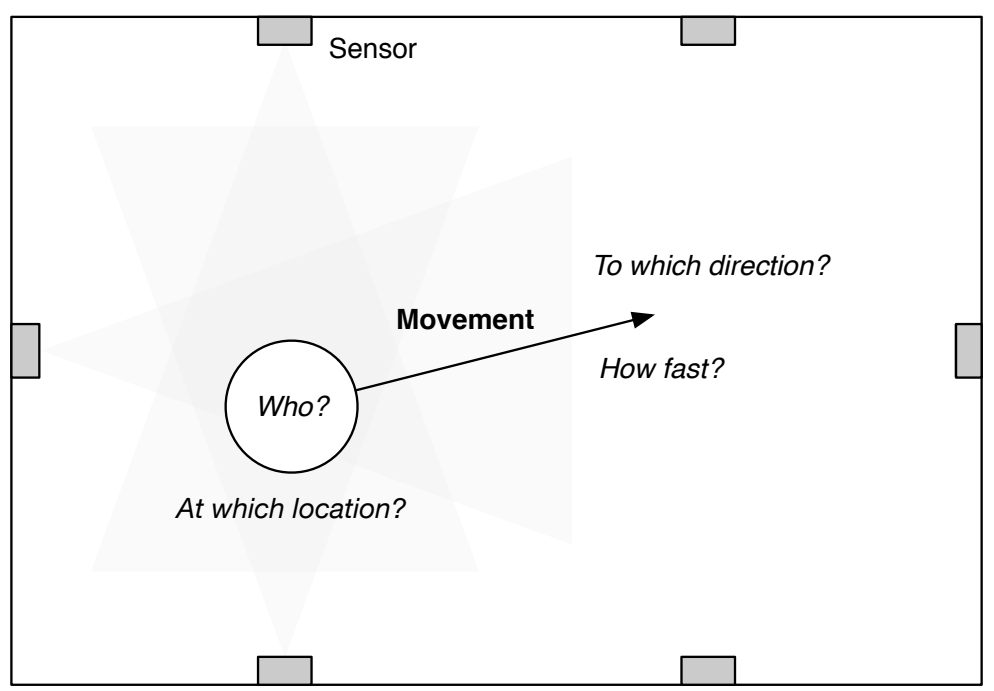

Figure 2. A schematic presentation of a pyroelectric infrared (PIR) sensor with dual sensing elements aligned in a motion plane and its output signal when walking: (a) the output signal in the case of walking in different directions; (b) the output signal in the case of walking at different distances; (c) the output signal in the case of walking at different speed levels.
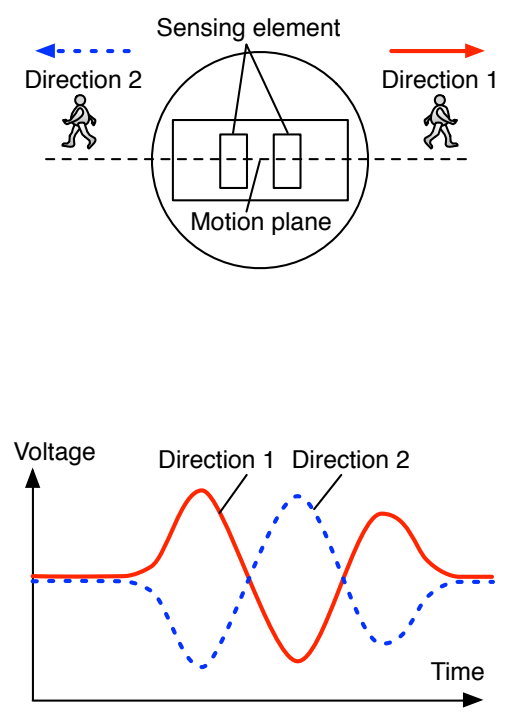

(a)
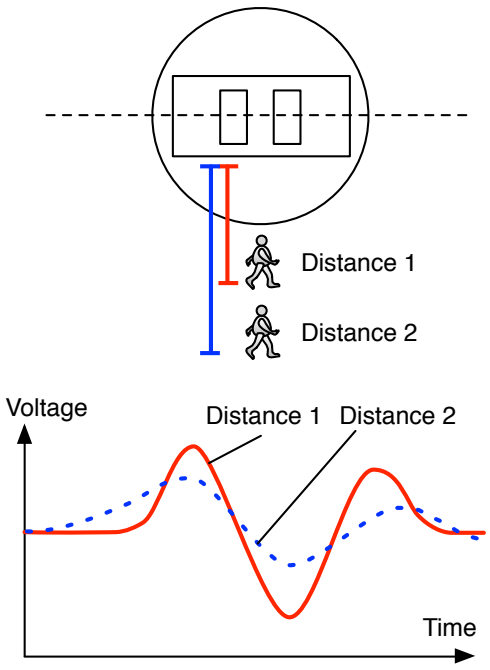

(b)
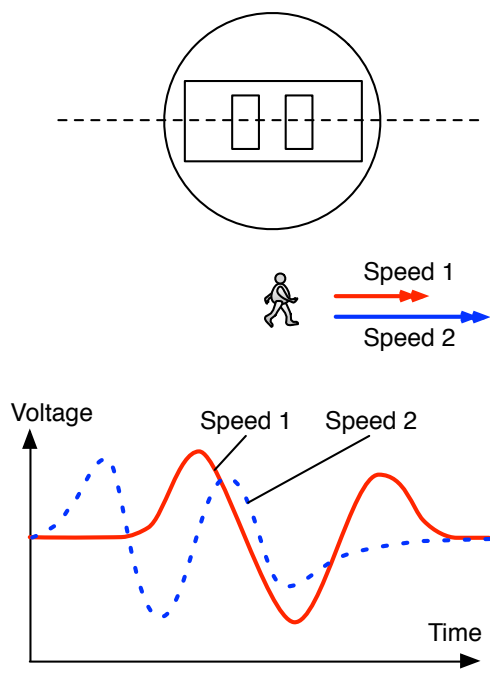

(c) 


\subsection{Pyroelectric Infrared Sensors}

Sensing systems for human movement detection and identification collect a raw data set from the human body and extract distinguishable features to recognize the principal context that we mentioned previously: the object location, the direction of the movement, the speed of the movement and the identity of the object. Numerous sensing systems have been studied using various sensors, including cameras, motion sensors, pressure pads, radars, electric field sensors. Among them, in this paper, we focus on pyroelectric infrared (PIR) sensors as sensing systems to detect human body movement in an indoor environment.

PIR sensors are well known and have been widely used as a simple, but powerful presence trigger for alarms, such as surveillance systems and automatic lighting control. In particular, PIR sensors could address the invasion of privacy issues raised by the use of camera-based surveillance systems. Whatmore presented an extensive survey on the technology of pyroelectric materials, devices and its potential applications [24]. PIR sensors belong to the class of thermal IR detectors, i.e., voltage change-thermoelectrical conversion, and use materials having a pyroelectric effect, e.g., $\mathrm{LiTaO}_{3}$, which is spontaneously polarized in the crystal structure [25]. Although PIR sensors have been commonly used as simple presence triggers using digital output, the analog output signal of PIR sensors depends on several aspects, including the direction and speed of a moving object, the distance of the body from the PIR sensor, the body shape and the presence of multiple people.

Figure 2 is a schematic presentation of a PIR sensor having two sensing elements aligned in a motion plane and its output signal captured in the case of walking: (1) in different directions; (2) at different distances; and (3) at different speed levels. The graph from Figure 2a shows that by alternating the polarities of the sensing elements in a PIR sensor, we can discriminate between the two signals captured according to the relative directions of walking, i.e., left to right and right to left, and subsequently to obtain the clear direction information of the moving object. Similarly, the graphs from Figure $2 b, c$ show that by using the different output signal patterns (e.g., amplitude and time to peaks), we can discriminate between the two signals captured according to the distances of the human body from the PIR sensor and the speed levels of the walking human, i.e., close/far and slow/fast; and subsequently, to obtain the clear distance and speed information of the moving object. Finally, with all these aspects, we can imagine a way of identifying the moving object based on a human movement detecting device composed of a set of PIR sensors. Therefore, the main result of this paper is to demonstrate a way of detecting human movement and identifying subjects based on a human movement detecting device composed of a set of PIR sensors and to discuss the experimental results according to direction, distance and speed estimation for human movement and, finally, human identification.

\section{A PIR-Based Movement Detecting Device}

\subsection{PIR Module and DAQ System}

As Yun and Song proposed in [16], two pairs of PIR sensors whose sensing elements are orthogonally aligned could classify walking directions into eight categories (i.e., N, NW, W, SW, S, SE, E, NE). For our research, we also assume that the signals collected from the orthogonally-aligned PIR-based detecting 
device are good enough to distinguish between the distances of the human body from the PIR sensors and the speeds of the moving body.

Accordingly, for our experiments, we have created a human movement detecting device based on two pairs of PIR sensors orthogonally aligned, as shown in Figure 3. The human movement detecting device consists of two pairs of PIR sensors, a Logomatic v2 Serial SD data logger from SparkFun Electronics, a rechargeable battery and a custom-fabricated data acquisition board. Figure 3 a shows a schematic diagram of a set of four PIR sensors whose sensing elements are each oriented at W-E, NW-SE, S-N and SW-NE angles, and Figure $3 \mathrm{~b}$ is a top view of the four PIR sensors we have implemented. PIR sensors are separated by a $2-\mathrm{cm}$ space considering the Fresnel lens setup, which is able to shape the field of view of PIR sensors. We have used IRA-E710 PIR sensors from Murata Manufacturing Co., whose vertical and horizontal field of views are both $90^{\circ}$ [26]. Because IRA-E710 PIR sensors output a low analog voltage signal, we have developed op-amp circuits to amplify the low PIR output signal. To acquire amplified PIR sensor signals, we have created a printed circuit board (PCB) having PIR sensors and op-amp circuits, whose outputs are connected to the analog inputs of a Logomatic v2 Serial SD data logger. The Logomatic v2 SD data logger is configured to record each analog input as a time series on a 2 GB micro-SD memory card, with a sampling rate of $10 \mathrm{~Hz}$. It is already known that a $10-\mathrm{Hz}$ sampling rate shows the best performance in human activity analysis based on PIR sensor-based systems [21]. A rechargeable battery can continuously power four PIR sensors and the data logger during data collection. We can also power the data collection module with two AA batteries.

Figure 3. (a) A schematic diagram of a set of four PIR sensors whose sensing elements are each oriented at W-E, NW-SE, S-N and SW-NE angles; (b) a top view of four PIR sensors we implemented; (c) a top view of the human movement detecting device consisting of PIR sensors, a Logomatic v2 Serial SD data logger from SparkFun Electronics, a rechargeable battery and a custom-fabricated data acquisition board integrated with op-amp circuits.

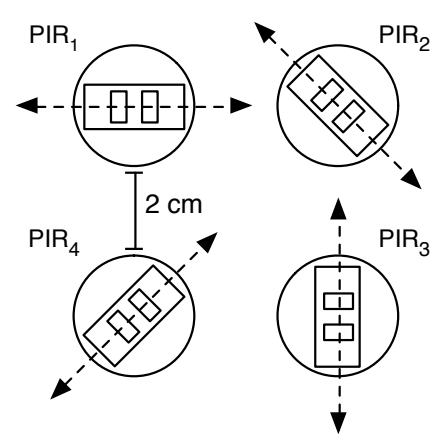

(a)

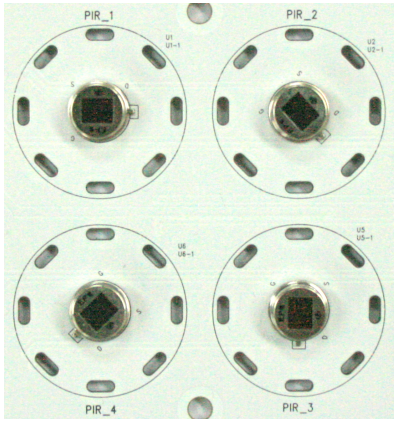

(b)

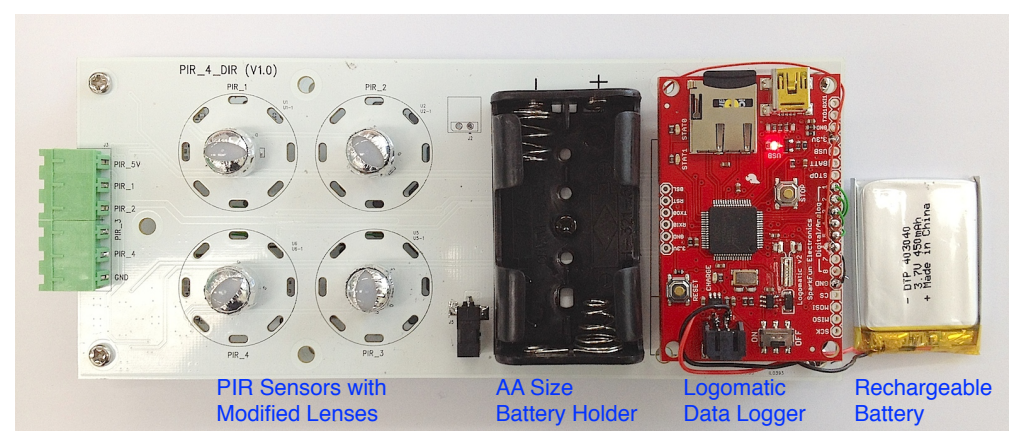

(c) 
PIR sensors are used in conjunction with Fresnel lenses to shape their field of view [27]. Fresnel lenses can be manufactured by molding plastic materials having transmission characteristics appropriate for a particular wavelength range, e.g., the human body $(8-14 \mu \mathrm{m})$. For our experiments, instead of using multi-zone Fresnel lenses mainly employed in traditional intruder detection systems [28], we used single-zone Fresnel lenses, IML-0637, from Murata Manufacturing Co. [26]. As presented in [16], we assume that by narrowing the field of view of PIR sensors to its horizontal motion plane aligned with the sensing elements, we could reduce the signal captured from walking in the other directions, and this would help in distinguishing the walking direction aligned with the sensing elements from the other directions, thus improving recognition accuracy. With the assumption, we have shielded IML-0637, single-zone Fresnel lenses with metallic foils, as shown in Figure 3c.

\subsection{Experimental Setup and Data Collection}

Our experiments consisted of capturing PIR sensor signals while experimental subjects are walking through a monitored field. A hallway in our office building is chosen as a monitoring field for collecting PIR sensor signals. Of course, office rooms or larger public areas could be considered, but the PIR sensor-based human movement detecting device mounted on walls (probably, the ceiling-mounted one is good for collecting data sets) may not collect data sets appropriate for our analysis due to the long distance of the body from the PIR sensor. Accordingly, we selected an indoor scenario, i.e., monitoring people walking through a hallway in our building. The hallway is $2.1 \mathrm{~m}$ wide, and the ceiling is $2.6 \mathrm{~m}$ high. For data collection, we have placed three PIR-based modules that we developed in the hallway; one module on the ceiling; two modules on opposite walls facing each other, as shown in Figure 4a. The ceiling-mounted PIR-based module is attached to the ceiling in the middle of the field. Two wall-mounted PIR-based modules are attached on opposite walls facing each other, at a height of $0.8 \mathrm{~m}$ from the floor. It has been already shown that a set of PIR sensors and Fresnel lenses located at a height of $0.8 \mathrm{~m}$ presents the best performance for path-dependent human identification [18].

Figure 4. Experimental setup for data collection while an experimental subject is walking, (a) a front view of the monitored field; (b) a top view of the monitored field.

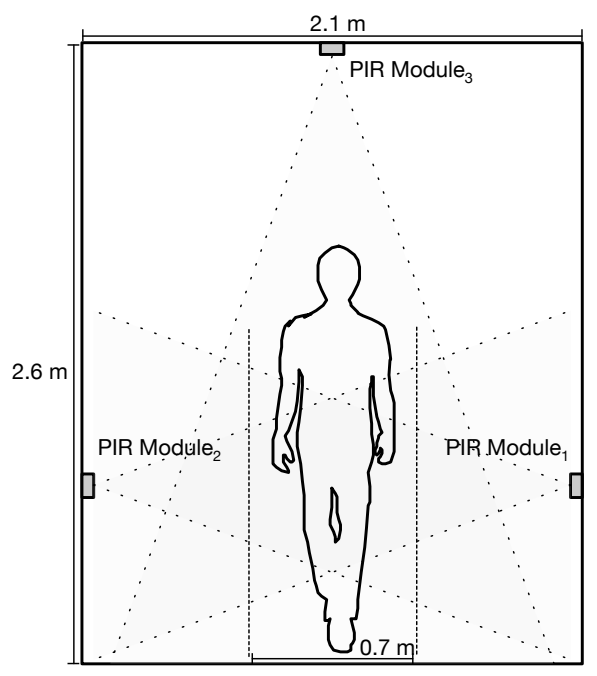

(a)

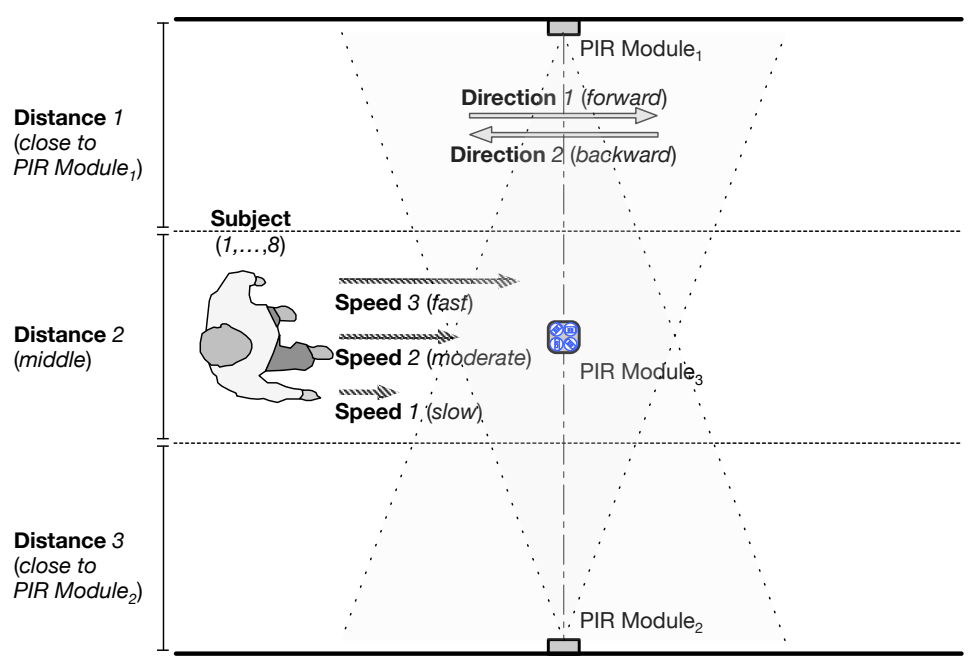

(b) 
We have collected PIR sensor signals from eight experimental subjects. The average height of the subjects is $170.67 \mathrm{~cm}$, and the standard deviation is $5.22 \mathrm{~cm}$. The average weight of the subjects is $69.22 \mathrm{~kg}$, and the standard deviation is $8.32 \mathrm{~kg}$. Each participant is asked to walk through the monitored field equipped with three PIR-based modules in three different conditions: direction, walking in two different directions (i.e., forward and backward); distance, walking at three different distances from PIR modules (i.e., close to PIR Module ${ }_{1}$, middle, close to PIR Module ${ }_{2}$ ); speed, walking at three different speed levels (i.e., slow, moderate, fast), as shown in Figure 4b. All subjects are asked to walk as normally as possible within the range of their daily walking style. For each experimental condition-direction, distance, speed-we have captured 10 walking samples from each participant and, finally, collected a total of $1,440(=8$ subjects $\times 2$ directions $\times 3$ distance intervals $\times 3$ speed levels $\times 10$ passages $)$ walking samples.

\subsection{Data Analysis and Feature Extraction}

Figure 5 shows the signals captured from a PIR sensor (i.e., $\mathrm{PIR}_{1}$ in Figure 3) of the wall-mounted data collection module (i.e., PIR Module ${ }_{1}$ in Figure 4) and a photo of an experiment for data collection. Figure 5a-c shows the captured output signals of a PIR sensor in the case of different experimental conditions, i.e.: (1) walking forward and backward; (2) walking at three different distance intervals (close, middle, far); (3) and walking at three different speed levels (slow, moderate, fast), respectively. From each figure, we can know that the captured PIR signals vary according to the different experimental conditions, as we explained in Figure 2. Accordingly, we will be able to use these quite different patterns of the PIR output signals in the case of different experimental conditions and leverage them to detect the aspects of human movement, including direction, distance interval and speed level, and, finally, identify the subject.

Because it is clear to see that there are quite different patterns between PIR output signals captured in different experimental conditions, we could employ a raw data set consisting of the time series captured from the PIR-based modules as a feature set for classifying directions, distances and speeds and identifying experimental subjects. In this case, assuming that we use all four PIR sensors of each of the three PIR-based modules, and a walking sample is $3 \mathrm{~s}$, a feature set for a walking sample is composed of 360 amplitude values (= 3 PIR-based modules $\times 4$ PIR sensors/PIR-based module $\times 3 \mathrm{~s} \times$ 10 values/s).

However, it is already known that the signal amplitude (the difference between the maximum and minimum value of the PIR output) and the passage duration (the time during the PIR output exceeds custom thresholds) can be used as a feature set to classify the distance of movement from the PIR sensors [15]. They also used a peak detection method (one positive and one negative) to recognize the direction of movement. It is also presented that the amplitude values of three peaks in PIR signal can be used as a feature set for classifying walking directions [16]. Accordingly, as shown in Figure 6, we have decided to employ a reduced feature set consisting of amplitude $\left(A_{1}, A_{2}, A_{3}\right)$ and time $\left(T_{1}, T_{2}, T_{3}\right)$ to three peaks and passage duration (D) for a PIR sensor for our classification analysis. In this case, assuming that we use all four PIR sensors of each of three PIR-based modules, a feature set 
for a walking sample is composed of 84 features (= 3 PIR-based modules $\times 4$ PIR sensors/PIR-based module $\times 7$ features/PIR sensor).

Figure 5. The output signals captured from the $\mathrm{PIR}_{1}$ sensor of the wall-mounted Module in the case of different experimental conditions, and a photo of an experiment for data collection: (a) the PIR output signal in the case of walking forward and backward; (b) the PIR output signal in the case of walking at three different distances; (c) the PIR output signal in the case of walking at three different speed levels; (d) an experiment for collecting PIR signals when walking.

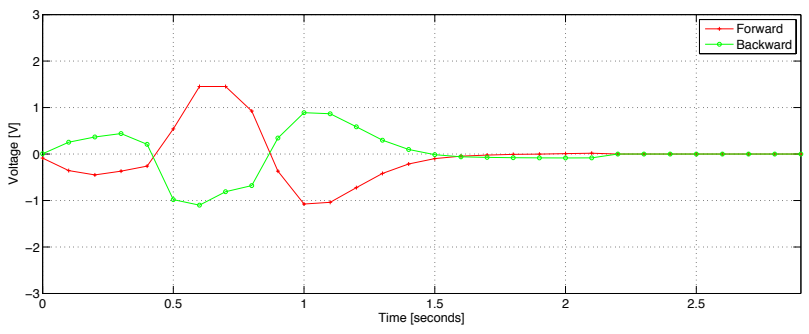

(a)

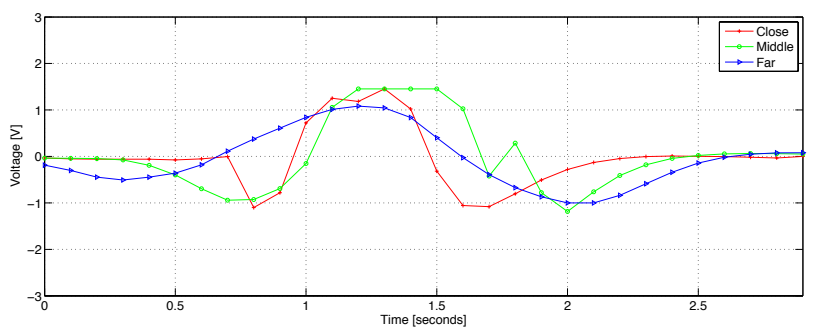

(b)

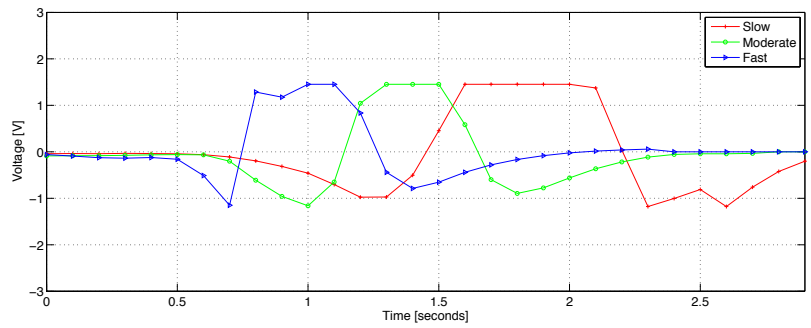

(c)

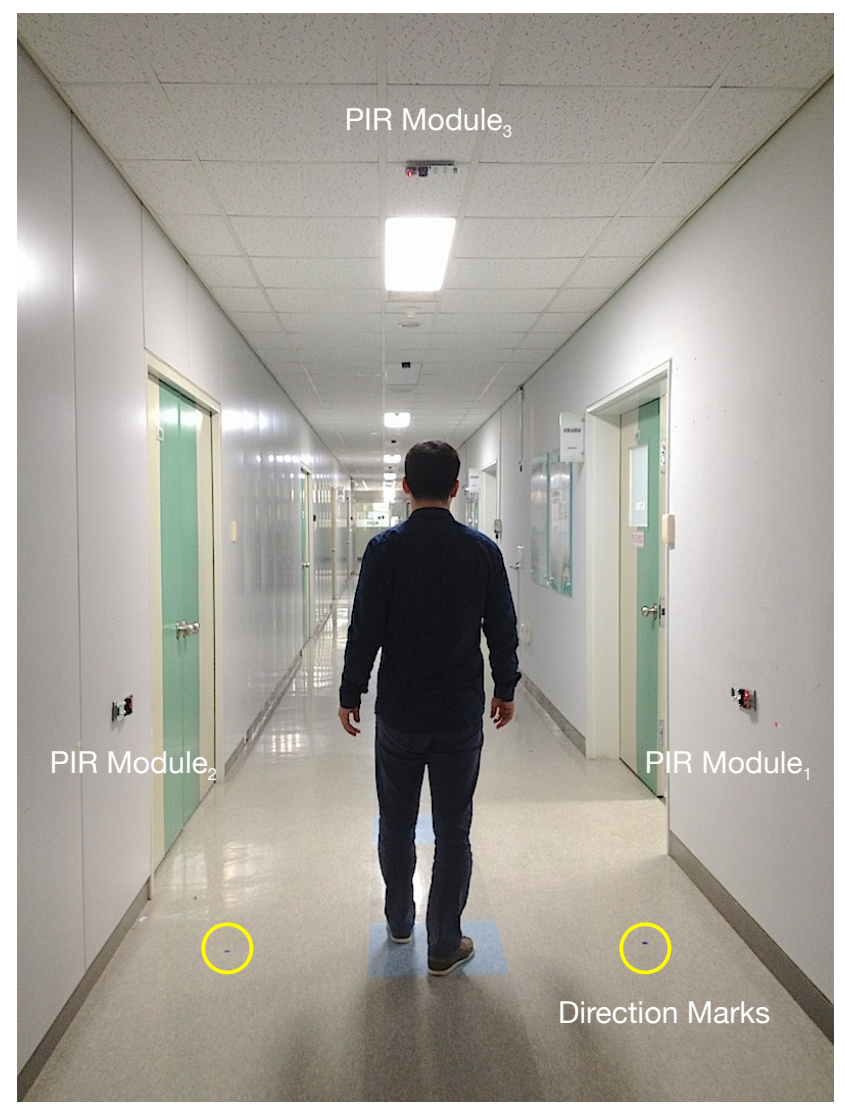

(d)

Figure 6. Feature extraction, amplitude $\left(A_{1}, A_{2}, A_{3}\right)$ and time $\left(T_{1}, T_{2}, T_{3}\right)$ to three peaks and passage duration (D).

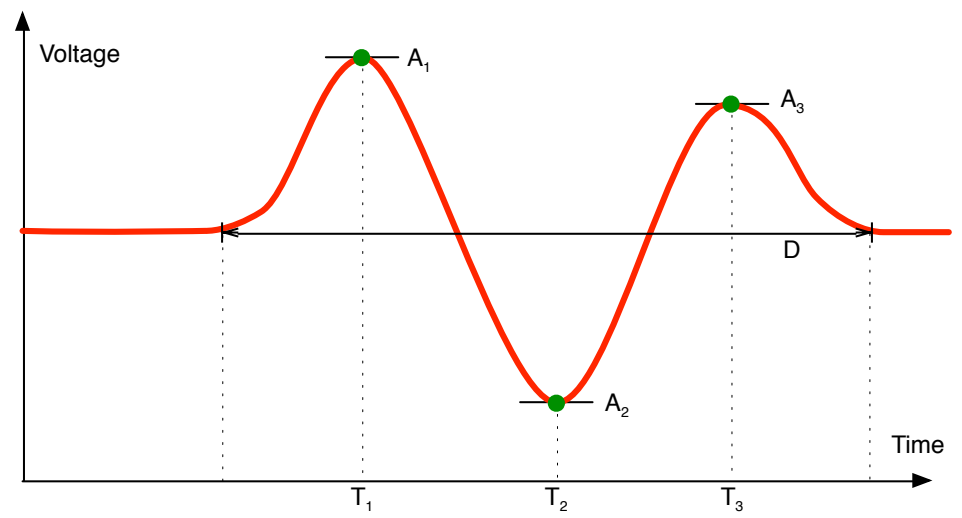


Even though we can build a reduced feature set for our classification analysis, we might be able to further reduce features by removing redundant PIR sensors or modules. That is, without employing all three PIR-based modules or all four PIR sensors in each module, we might be able to achieve reasonable performance for classification analysis, finally reducing the computational cost and memory requirement for training and classifying data sets. We will demonstrate the effect of the trade-off between the amount of computation load and recognition accuracy in the result sections below.

\subsection{Classifiers}

Among various available machine learning algorithms, we chose seven classification methods: Bayes net, decision tree (C4.5), decision table, instance-based learning ( $k$-nearest neighbor algorithm), multilayer perceptron, naive Bayes and support vector machine. Support vector machine is chosen as one of the state-of-the-art discriminative methods with a good performance in many applications. For support vector machine, we use three types of kernels, including linear, quadratic and cubic. We chose the simple $k$-nearest neighbor algorithm from instance-based learning algorithms and the decision tree and decision table from rule-based learning algorithms. In addition, Bayes net is chosen as one of generative models to show its performance in our experiments. We also used naive Bayes and multilayer perceptron as classifiers. All the experiments based on these classifiers were carried out using Weka developed by the Machine Learning Group at University of Waikato [29]. Weka is a data mining tool with open source machine learning software in Java. It supports all the classification methods we chose for classification. Therefore, we would be able to compare the experimental results among various classifiers and feature sets. We also used Eclipse to build and run a Java program based on the Java classes supported by Weka [30].

\section{Experiments and Results}

In our experiments, we used the 1,440 walking samples collected from eight subjects in different walking conditions, i.e., two different directions (back and forth), three different speed levels (slow, moderate and fast) and three different distance intervals (close to one sensor, in the middle, close to the other sensor), as explained in Section 4.2. For each experiment, we used all 1,440 walking samples combined with 10 times 10-fold cross-validation, in other words, 10 different 10-fold cross-validation experiments with the same learning method and data set, averaging 100 experimental results. In the experimental result, we summarize the average recognition accuracy, but we do not show the standard deviation of the results, because the average values are very close to each other (i.e., very small standard deviation) for all classifiers. We have performed classification analysis with two types of feature sets: raw data set and reduced feature set. In Section 5.1, we first present the experimental result with the raw data set, i.e., the time series captured from the PIR-based modules for classifying walking direction, distance and speed and identifying subjects; and, then, in Section 5.2, we demonstrate the classification result with the reduced feature set explained in Section 4.3, composed of amplitude and time to peak values and passage duration extracted from the PIR sensor signals. 


\subsection{Classification Analysis with the Raw Data Set}

In this section, we demonstrate the experimental result with the raw data set, i.e., the time series captured from the PIR-based modules for classifying walking directions (backward and forward), distance intervals (close, middle, far), speed levels (slow, moderate, fast) and identifying subjects.

\subsubsection{Classifying Directions with the Raw Data Set}

Table 1 summarizes the experimental result for classifying walking directions (i.e., forward and backward) over the selected classification methods based on PIR signals. In Table 1, we can know that the instance-based learning method ( $k$-nearest neighbor algorithm) and Bayes net show the best classification performance. This result is not surprising, because the past works in $[15,16]$ performed the recognition of motion direction with the $k$-nearest neighbor algorithm and show a good recognition accuracy. Support vector machine, in particular, with linear kernel and multilayer perceptron, also show good performance. The other algorithms show almost or over $98 \%$ recognition accuracy, except naive Bayes.

Table 1. Comparison of recognition accuracy (\%) of classifying walking directions based on the raw data set with respect to the number of PIR sensors of all modules.

\begin{tabular}{|c|c|c|c|}
\hline \multirow{5}{*}{ Classifier } & \multicolumn{3}{|c|}{ Recognition Accuracy (\%) } \\
\hline & \multicolumn{3}{|c|}{$\begin{array}{c}\text { Module }_{1,2,3} \\
\square\end{array}$} \\
\hline & \multicolumn{3}{|c|}{$\square \quad \square$} \\
\hline & $\mathrm{PIR}_{1}$ & $\mathrm{PIR}_{1,3}$ & $\mathrm{PIR}_{1,2,3,4}$ \\
\hline & YY & & \\
\hline Bayes Net & 99.99 & 99.99 & 99.99 \\
\hline Decision Table & 97.72 & 97.74 & 98.35 \\
\hline Decision Tree & 97.41 & 97.76 & 98.95 \\
\hline Instance-based Learning & 99.99 & 99.98 & 99.99 \\
\hline Multilayer Perceptron & 99.84 & 99.88 & 99.93 \\
\hline Naive Bayes & 84.15 & 85.76 & 92.78 \\
\hline SVM (linear kernel) & 99.85 & 99.79 & 99.95 \\
\hline SVM (quadratic kernel) & 99.75 & 99.80 & 99.95 \\
\hline SVM (cubic kernel) & 99.72 & 99.80 & 99.91 \\
\hline
\end{tabular}

Note that classifying directions with a single $\left(\mathrm{PIR}_{1}\right)$ sensor of each of all three modules performs as well as the other cases, i.e., using a pair of PIR sensors orthogonally aligned ( $\mathrm{PIR}_{1}$ and $\mathrm{PIR}_{3}$ ) or two pairs of PIR sensors orthogonally aligned $\left(\mathrm{PIR}_{1}\right.$ and $\mathrm{PIR}_{3}, \mathrm{PIR}_{2}$ and $\left.\mathrm{PIR}_{4}\right)$. Therefore, we can know that a single PIR sensor of each PIR-based module will be enough to achieve a good performance in the two-way back-and-forth walking direction recognition. Now, we need to perform an investigation into the recognition performance with respect to the number of modules involved.

Table 2 summarizes the experimental result for classifying walking directions with respect to the number of modules involved based on PIR 1 signals. The result with two wall-mounted PIR-based modules facing each other (i.e., Module ${ }_{1}$ and Module $_{2}$ ) shows $100 \%$ correct detection of walking direction, as also presented in [15]. More surprisingly, the results with a single module, i.e., a single PIR sensor on the ceiling or walls, performs more than a $99 \%$ correct detection of the back-and-forth 
walking direction, probably because the $\mathrm{PIR}_{1}$ sensor of every module (and, thus, the sensing elements in the PIR sensor) is aligned with the motion plane of walking.

Table 2. Comparison of the recognition accuracy (\%) of classifying walking directions based on the raw data set $\left(\mathrm{PIR}_{1}\right)$ with respect to the number of modules.

\begin{tabular}{|c|c|c|c|c|c|c|c|}
\hline \multirow{6}{*}{ Classifier } & \multicolumn{7}{|c|}{ Recognition Accuracy (\%) } \\
\hline & $\underset{\times}{\text { Module }_{1}}$ & $\underset{\times}{\text { Module }_{2}}$ & $\begin{array}{c}\text { Module }_{3} \\
\square\end{array}$ & $\underset{\times}{\text { Module }_{1,2}}$ & $\begin{array}{c}\text { Module }_{1,3} \\
\square\end{array}$ & $\begin{array}{c}\text { Module }_{2,3} \\
\square\end{array}$ & $\begin{array}{c}\text { Module }_{1,2,3} \\
\square\end{array}$ \\
\hline & $\times \quad \square$ & $\square \quad \times$ & $\times \quad \times$ & $\square \quad \square$ & $\times \quad \square$ & $\square \quad \times$ & $\square \quad \square$ \\
\hline & $\mathrm{PIR}_{1}$ & $\mathrm{PIR}_{1}$ & $\mathrm{PIR}_{1}$ & $\mathrm{PIR}_{1}$ & $\mathrm{PIR}_{1}$ & $\mathrm{PIR}_{1}$ & $\mathrm{PIR}_{1}$ \\
\hline & $O \times$ & $O x$ & $O \times$ & $O x$ & $O \times$ & $O \times$ & $O x$ \\
\hline & $\times \times$ & $\times \times$ & $\times \times$ & $\times x$ & $x \times$ & $x \times$ & $\times \times$ \\
\hline Bayes Net & 99.51 & 98.58 & 95.43 & 100 & 99.41 & 99.75 & 99.99 \\
\hline Decision Table & 93.39 & 93.24 & 95.51 & 95.50 & 95.61 & 97.22 & 97.72 \\
\hline Decision Tree & 95.71 & 96.03 & 97.80 & 97.89 & 97.18 & 98.19 & 97.41 \\
\hline Instance-based Learning & 99.36 & 99.42 & 99.64 & 100 & 99.63 & 99.61 & 99.99 \\
\hline Multilayer Perceptron & 99.12 & 98.42 & 99.27 & 99.84 & 99.13 & 99.11 & 99.84 \\
\hline Naive Bayes & 86.03 & 85.52 & 63.31 & 73.50 & 83.25 & 65.22 & 84.15 \\
\hline SVM (linear kernel) & 93.23 & 92.00 & 74.12 & 99.76 & 98.49 & 98.61 & 99.85 \\
\hline SVM (quadratic kernel) & 97.86 & 96.86 & 90.63 & 99.65 & 98.97 & 98.79 & 99.75 \\
\hline SVM (cubic kernel) & 98.35 & 97.47 & 98.21 & 99.60 & 98.84 & 98.66 & 99.72 \\
\hline
\end{tabular}

\subsubsection{Classifying Distances with the Raw Data Set}

Table 3 summarizes the experimental result for classifying walking distances (i.e., close to PIR Module $_{1}$, middle, close to PIR Module 2 ). In Table 3, we can know that instance-based learning method ( $k$-nearest neighbor algorithm) and Bayes net show the best classification performance. This is very similar to the result obtained in walking direction classification and presented in the past work of [15]. Support vector machine, in particular, with quadratic and cubic kernels and multilayer perceptron, also show good performance, though those algorithms may require more computational load for training data sets than the $k$-nearest neighbor algorithm.

Table 3. Comparison of the recognition accuracy (\%) of classifying walking distance intervals based on the raw data set with respect to the number of PIR sensors of all modules.

\begin{tabular}{cccc}
\hline & \multicolumn{3}{c}{ Recognition Accuracy (\%) } \\
\cline { 2 - 4 } Classifier & \multicolumn{3}{c}{ Module $_{1,2,3}$} \\
& $\mathrm{PIR}_{1}$ & $\mathrm{PIR}_{1,3}$ & $\mathrm{PIR}_{1,2,3,4}$ \\
& $\bigcirc \times$ & $\bigcirc \times$ & $\bigcirc \bigcirc$ \\
\cline { 2 - 4 } & $\times \times$ & $\times \bigcirc$ & 99.93 \\
\hline Bayes Net & $\mathbf{9 9 . 8 2}$ & 99.86 & 97.50 \\
\hline Decision Table & 95.50 & 97.50 & 98.78 \\
\hline Decision Tree & 97.68 & 98.72 & 99.81 \\
\hline Instance-based Learning & $\mathbf{9 9 . 5 2}$ & 99.66 & 99.61 \\
\hline Multilayer Perceptron & 97.81 & 99.48 & 97.41 \\
\hline Naive Bayes & 95.00 & 96.88 & 99.57 \\
\hline SVM (linear kernel) & 88.48 & 94.29 & 99.76 \\
\hline SVM (quadratic kernel) & 99.13 & 99.56 & \\
\hline SVM (cubic kernel) & 99.47 & 99.70 &
\end{tabular}


Similar to the result of walking direction classification, a single PIR sensor of each PIR-based module will be enough to achieve a good performance. Thus, we perform an investigation into the recognition performance with respect to the number of modules involved. Table 4 summarizes the experimental result for classifying walking distances with respect to the number of modules. The result with two wall-mounted PIR-based modules facing each other (i.e., Module ${ }_{1}$ and Module $_{2}$ ) shows about a 95\% correct detection of walking distance intervals. The results with a ceiling-mounted module (i.e., Module $_{3}$ ) show a performance of more than $97 \%$ correct detection of walking distance intervals (even better than two wall-mounted modules), thus increasing the recognition accuracy (>99\%) when using all three PIR-based modules, including wall- and ceiling-mounted modules.

Table 4. Comparison of the recognition accuracy (\%) of classifying the walking distance intervals based on the raw data set $\left(\mathrm{PIR}_{1}\right)$ with respect to the number of modules.

\begin{tabular}{|c|c|c|c|c|c|c|c|}
\hline \multirow{6}{*}{ Classifier } & \multicolumn{7}{|c|}{ Recognition Accuracy (\%) } \\
\hline & $\begin{array}{c}\text { Module }_{1} \\
\times\end{array}$ & $\underset{\times}{\text { Module }_{2}}$ & $\begin{array}{c}\text { Module }_{3} \\
\square\end{array}$ & Module $_{1,2}$ & $\begin{array}{c}\text { Module }_{1,3} \\
\square\end{array}$ & $\begin{array}{c}\text { Module }_{2,3} \\
\square\end{array}$ & $\begin{array}{c}\text { Module }_{1,2,3} \\
\square\end{array}$ \\
\hline & $\times \quad \square$ & $\square \quad \times$ & $\times \quad \times$ & $\square \quad \square$ & $\times \quad \square$ & $\square \quad \times$ & $\square \quad \square$ \\
\hline & $\mathrm{PIR}_{1}$ & $\mathrm{PIR}_{1}$ & $\mathrm{PIR}_{1}$ & $\mathrm{PIR}_{1}$ & $\mathrm{PIR}_{1}$ & $\mathrm{PIR}_{1}$ & $\mathrm{PIR}_{1}$ \\
\hline & $O x$ & $O x$ & $O \times$ & $\bigcirc \times$ & $O \times$ & $O x$ & $O \times$ \\
\hline & $\times \times$ & $x \times$ & $x \times$ & $x \times$ & $x \times$ & $x \times$ & $\times \times$ \\
\hline Bayes Net & 75.56 & 69.44 & 95.39 & 73.46 & 99.48 & 98.22 & 98.82 \\
\hline Decision Table & 72.95 & 70.45 & 92.27 & 78.73 & 93.82 & 94.92 & 95.50 \\
\hline Decision Tree & 85.94 & 84.07 & 95.06 & 87.13 & 97.92 & 98.04 & 97.68 \\
\hline Instance-based Learning & 81.92 & 79.63 & 97.68 & 95.51 & 97.89 & 96.04 & 99.52 \\
\hline Multilayer Perceptron & 72.88 & 69.65 & 74.36 & 81.02 & 97.35 & 97.54 & 97.81 \\
\hline Naive Bayes & 53.40 & 53.04 & 93.91 & 63.20 & 93.72 & 86.13 & 95.00 \\
\hline SVM (linear kernel) & 46.27 & 44.75 & 45.69 & 59.08 & 71.47 & 86.78 & 88.48 \\
\hline SVM (quadratic kernel) & 75.45 & 73.14 & 70.15 & 95.78 & 97.97 & 97.15 & 99.13 \\
\hline SVM (cubic kernel) & 83.34 & 80.11 & 75.40 & 95.93 & 98.24 & 97.01 & 99.47 \\
\hline
\end{tabular}

\subsubsection{Classifying Speeds with the Raw Data Set}

Table 5 summarizes the experimental result for classifying walking speed levels (i.e., slow, moderate, fast). In Table 5, we can know that the instance-based learning method ( $k$-nearest neighbor algorithm) shows again the best classification performance. Support vector machine, in particular, with the quadratic and cubic kernels, also shows good performance for walking speed classification.

Similar to the result of classifying walking directions and distance intervals, a single PIR sensor of each PIR-based module will be enough, and thus, we perform classification analysis with respect to the number of modules involved. Table 6 summarizes the experimental result for classifying walking speed levels with respect to the number of modules. The result with two wall-mounted PIR-based modules facing each other (i.e., Module ${ }_{1}$ and Module $_{2}$ ) shows about $92 \%$ correct detection of walking speed levels. The result with a ceiling-mounted module (i.e., Module ${ }_{3}$ ) performs more than a $90 \%$ correct detection of walking speed, thus increasing the recognition accuracy (about 95\%) when using all three PIR-based modules, including wall- and ceiling-mounted modules. 
Table 5. Comparison of the recognition accuracy (\%) of classifying walking speed levels based on the raw data set with respect to the number of PIR sensors of all modules.

\begin{tabular}{cccc}
\hline & \multicolumn{3}{c}{ Recognition Accuracy (\%) } \\
\cline { 2 - 4 } Classifier & \multicolumn{3}{c}{ Module $_{1,2,3}$} \\
& \multicolumn{4}{c}{} \\
\cline { 2 - 4 } & $\mathrm{PIR}_{1}$ & $\mathrm{PIR}_{1,3}$ & $\mathrm{PIR}_{1,2,3,4}$ \\
& $\times \times$ & $\times$ & $\bigcirc$ \\
\hline Bayes Net & $\times \times$ & 79.11 \\
\hline Decision Table & 77.93 & 78.34 & 77.08 \\
\hline Decision Tree & 89.59 & 76.83 & 89.34 \\
\hline Instance-based Learning & $\mathbf{9 5 . 4 3}$ & 89.04 & 95.87 \\
\hline Multilayer Perceptron & 92.44 & 95.72 & 93.29 \\
\hline Naive Bayes & 68.97 & 68.74 & 68.96 \\
\hline SVM (linear kernel) & 81.25 & 82.38 & 84.76 \\
\hline SVM (quadratic kernel) & 94.71 & 95.09 & 95.61 \\
\hline SVM (cubic kernel) & 95.15 & 95.68 & 96.49 \\
\hline
\end{tabular}

Table 6. Comparison of the recognition accuracy (\%) of classifying walking speed levels based on the raw data set $\left(\mathrm{PIR}_{1}\right)$ with respect to the number of modules.

\begin{tabular}{|c|c|c|c|c|c|c|c|}
\hline \multirow{6}{*}{ Classifier } & \multicolumn{7}{|c|}{ Recognition Accuracy (\%) } \\
\hline & $\underset{\times}{\text { Module }_{1}}$ & $\underset{\times}{\text { Module }_{2}}$ & $\begin{array}{c}\text { Module }_{3} \\
\square\end{array}$ & Module $_{1,2}$ & $\begin{array}{c}\text { Module }_{1,3} \\
\square\end{array}$ & $\begin{array}{c}\text { Module }_{2,3} \\
\square\end{array}$ & $\begin{array}{c}\text { Module }_{1,2,3} \\
\square\end{array}$ \\
\hline & $\times \quad \square$ & $\square \quad \times$ & $x$ & $\square \quad \square$ & $\times \quad \square$ & $\square \quad \times$ & $\square \quad \square$ \\
\hline & $\mathrm{PIR}_{1}$ & $\mathrm{PIR}_{1}$ & $\mathrm{PIR}_{1}$ & $\mathrm{PIR}_{1}$ & $\mathrm{PIR}_{1}$ & $\mathrm{PIR}_{1}$ & $\mathrm{PIR}_{1}$ \\
\hline & $O x$ & $O \times$ & $O \times$ & $O x$ & $O x$ & $O \times$ & $O \times$ \\
\hline & $x \times$ & $x \times$ & $x \times$ & $x \times$ & $\times \times$ & $x \times$ & $\times x$ \\
\hline Bayes Net & 67.31 & 70.24 & 78.94 & 75.83 & 77.31 & 77.77 & 77.93 \\
\hline Decision Table & 72.49 & 75.29 & 76.08 & 75.35 & 74.98 & 75.50 & 76.59 \\
\hline Decision Tree & 82.84 & 84.13 & 84.81 & 87.81 & 87.65 & 86.84 & 89.19 \\
\hline Instance-based Learning & 79.34 & 80.36 & 90.13 & 92.55 & 90.19 & 89.15 & 95.43 \\
\hline Multilayer Perceptron & 70.18 & 72.06 & 77.41 & 90.84 & 87.02 & 83.77 & 92.44 \\
\hline Naive Bayes & 62.24 & 61.73 & 50.25 & 66.79 & 66.76 & 63.64 & 68.97 \\
\hline SVM (linear kernel) & 50.63 & 58.95 & 56.99 & 71.87 & 74.67 & 71.87 & 81.25 \\
\hline SVM (quadratic kernel) & 72.18 & 74.15 & 76.11 & 95.29 & 92.21 & 89.63 & 94.71 \\
\hline SVM (cubic kernel) & 77.51 & 79.19 & 80.90 & 94.63 & 90.28 & 90.56 & 95.15 \\
\hline
\end{tabular}

\subsubsection{Identifying Subjects with the Raw Data Set}

Table 7 summarizes the experimental result for identifying subjects. In Table 7, we can know that the instance-based learning method ( $k$-nearest neighbor algorithm) outperforms all other algorithms and shows the best classification accuracy. Support vector machine with quadratic and cubic kernels is the second best algorithm.

Unlike previous classification analysis, such as distance, direction and speed, the more PIR sensors are involved, the better recognition accuracy could be achieved. However, the experimental result with a single ( $\left.\mathrm{PIR}_{1}\right)$ sensor of each of all three modules also shows a good performance (about 92\%); thus, this would be a trade-off between recognition accuracy and the computational load required. 
We also perform classification analysis with respect to the number of modules involved. Table 8 summarizes the experimental result for identifying walking subjects with respect to the number of modules. The result with two wall-mounted PIR-based modules facing each other (i.e., Module 1 and Module $_{2}$ ) shows about an $87 \%$ correct identification of walking subjects. The result with a ceiling-mounted module (i.e., Module $_{3}$ ) does not show a good performance (about 63\%), and thus, we can conclude that a human movement detecting system composed of only ceiling-mounted PIR sensors without wall-mounted ones may not identify walking subjects well.

Table 7. Comparison of the recognition accuracy (\%) of identifying walking subjects based on the raw data set with respect to the number of PIR sensors of all modules.

\begin{tabular}{cccc}
\hline & \multicolumn{3}{c}{ Recognition Accuracy (\%) } \\
\cline { 2 - 4 } Classifier & \multicolumn{3}{c}{ Module $_{1,2,3}$} \\
& $\mathrm{PIR}_{1}$ & $\square$ & $\mathrm{PIR}_{1,3}$ \\
& $\bigcirc \times$ & $\mathrm{PIR}_{1,2,3,4}$ & $\bigcirc$ \\
& $\times \times$ & $\times \bigcirc$ & $\bigcirc$ \\
\hline Bayes Net & 49.90 & 56.88 & 63.74 \\
\hline Decision Table & 48.18 & 52.87 & 51.41 \\
\hline Decision Tree & 72.38 & 76.15 & 76.27 \\
\hline Instance-based Learning & $\mathbf{9 2 . 6 5}$ & $\mathbf{9 4 . 3 9}$ & $\mathbf{9 5 . 2 0}$ \\
\hline Multilayer Perceptron & 57.38 & 65.86 & 69.86 \\
\hline Naive Bayes & 25.12 & 38.24 & 43.52 \\
\hline SVM (linear kernel) & 68.12 & 77.27 & 82.77 \\
\hline SVM (quadratic kernel) & 86.11 & 89.11 & 92.52 \\
\hline SVM (cubic kernel) & 88.43 & 91.13 & 94.14 \\
\hline
\end{tabular}

Table 8. Comparison of the recognition accuracy (\%) of identifying walking subjects based on the raw data set $\left(\mathrm{PIR}_{1}\right)$ with respect to the number of modules.

\begin{tabular}{|c|c|c|c|c|c|c|c|}
\hline \multirow{6}{*}{ Classifier } & \multicolumn{7}{|c|}{ Recognition Accuracy (\%) } \\
\hline & $\underset{\times}{\text { Module }_{1}}$ & $\underset{\times}{\text { Module }_{2}}$ & $\begin{array}{c}\text { Module }_{3} \\
\square\end{array}$ & $\underset{\times}{\text { Module }_{1,2}}$ & $\begin{array}{c}\text { Module }_{1,3} \\
\square\end{array}$ & $\begin{array}{c}\text { Module }_{2,3} \\
\square\end{array}$ & $\begin{array}{c}\text { Module }_{1,2,3} \\
\square\end{array}$ \\
\hline & $\square$ & & & $\square$ & $\square$ & $\square \times$ & $\square \quad \square$ \\
\hline & $\mathrm{PIR}_{1}$ & $\mathrm{PIR}_{1}$ & $\mathrm{PIR}_{1}$ & $\mathrm{PIR}_{1}$ & $\mathrm{PIR}_{1}$ & $\mathrm{PIR}_{1}$ & $\mathrm{PIR}_{1}$ \\
\hline & $O \times$ & $O \times$ & $O \times$ & $O x$ & $O x$ & $O x$ & $O \times$ \\
\hline & $x \times$ & $x \times$ & $x \times$ & $x \times$ & $\times x$ & $x \times$ & $\times x$ \\
\hline Bayes Net & 35.86 & 41.11 & 26.84 & 50.60 & 41.95 & 43.77 & 49.90 \\
\hline Decision Table & 35.41 & 37.40 & 28.40 & 48.91 & 42.98 & 44.88 & 48.18 \\
\hline Decision Tree & 55.33 & 57.84 & 52.91 & 72.24 & 67.92 & 67.86 & 72.38 \\
\hline Instance-based Learning & 59.55 & 55.09 & 63.07 & 87.30 & 77.43 & 74.59 & 92.65 \\
\hline Multilayer Perceptron & 29.57 & 31.68 & 23.82 & 50.46 & 45.69 & 42.22 & 57.38 \\
\hline Naive Bayes & 22.64 & 29.27 & 16.16 & 26.54 & 22.31 & 21.47 & 25.12 \\
\hline SVM (linear kernel) & 24.19 & 26.52 & 14.87 & 51.65 & 41.25 & 40.53 & 68.12 \\
\hline SVM (quadratic kernel) & 43.31 & 40.95 & 25.93 & 84.91 & 74.60 & 72.02 & 86.11 \\
\hline SVM (cubic kernel) & 56.36 & 52.58 & 39.56 & 86.15 & 75.22 & 70.95 & 88.43 \\
\hline
\end{tabular}

\subsection{Classification Analysis with the Reduced Feature Set}

In this section, we demonstrate the experimental result with the reduced feature set explained in Section 4.3, composed of amplitude and time to peaks and passage duration extracted from PIR sensor 
signals for classifying walking directions (backward and forward), distance intervals (close, middle, far), speed levels (slow, moderate, fast) and identifying subjects.

\subsubsection{Classifying Directions with the Reduced Feature Set}

In Section 5.1.1, we have presented a ceiling- or wall-mounted module equipped with a single $\mathrm{PIR}_{1}$ sensor can detect two-way, back-and-forth walking directions with more than $99 \%$ accuracy. We have also demonstrated the algorithms appropriate for walking direction classification, including Bayes net, instance-based learning, multilayer perceptron and support vector machine with a linear kernel. Accordingly, in this section, we present the experimental result of classifying walking directions with the reduced feature set and the machine learning algorithms that have shown a good performance in the experiments based on the raw data sets. Table 9 summarizes the experimental result for classifying walking directions based on the reduced feature sets with respect to the modules and features, such as amplitude and time to peaks. Regardless of the classification algorithms involved, the results with amplitude values without time information show more than $97 \%$ accuracy, except when using only the ceiling-mounted module, Module ${ }_{3}$. This is probably because narrowing the field of view of $\mathrm{PIR}_{1}$ by aluminum foil to its horizontal motion plane aligned with the sensing elements may reduce the signal captured (i.e., the amplitude of peaks), in particular, when the subjects are walking within Distance 1 or 3 (see Figure 4). Accordingly, we can conclude that for walking direction classification, a single PIR sensor placed on a wall and its amplitude values of peaks will be enough to achieve a good performance, but in case of a ceiling-mounted PIR sensor, we need to use both amplitude and time to peaks.

Table 9. Comparison of recognition accuracy (\%) of classifying walking directions based on the reduced feature set with respect to the modules and features (amplitude and time to peaks).

\begin{tabular}{|c|c|c|c|c|c|c|c|c|}
\hline \multirow{7}{*}{ Classifier } & \multicolumn{8}{|c|}{ Recognition Accuracy (\%) } \\
\hline & \multicolumn{2}{|c|}{$\underset{\times}{\text { Module }_{1}}$} & \multicolumn{2}{|c|}{$\underset{\times}{\text { Module }_{2}}$} & \multicolumn{2}{|c|}{$\begin{array}{c}\text { Module }_{3} \\
\square\end{array}$} & \multicolumn{2}{|c|}{ Module $_{1,2}$} \\
\hline & \multicolumn{2}{|c|}{$\begin{array}{l}\times \\
\times \quad \square\end{array}$} & \multicolumn{2}{|c|}{$\square \quad \times$} & & \multicolumn{2}{|c|}{$\square \quad \square$} \\
\hline & \multirow{3}{*}{\multicolumn{2}{|c|}{$\mathrm{PIR}_{1}$}} & \multirow{2}{*}{\multicolumn{2}{|c|}{$\begin{array}{l}\mathrm{PIR}_{1} \\
\bigcirc \times\end{array}$}} & \multirow{2}{*}{\multicolumn{2}{|c|}{$\begin{array}{l}\mathrm{PIR}_{1} \\
\bigcirc \times\end{array}$}} & \multicolumn{2}{|c|}{$\mathrm{PIR}_{1}$} \\
\hline & & & & & & & \multirow{2}{*}{\multicolumn{2}{|c|}{$\times \times$}} \\
\hline & & $\times \times$ & \multicolumn{2}{|c|}{$\times \times$} & \multicolumn{2}{|c|}{$\times \times$} & & \\
\hline & Amplitude & $\begin{array}{l}\text { Amplitude } \\
\text { and time }\end{array}$ & Amplitude & $\begin{array}{l}\text { Amplitude } \\
\text { and time }\end{array}$ & Amplitude & $\begin{array}{l}\text { Amplitude } \\
\text { and time }\end{array}$ & Amplitude & $\begin{array}{l}\text { Amplitude } \\
\text { and time }\end{array}$ \\
\hline Bayes Net & 97.50 & 97.77 & 96.15 & 97.27 & 77.77 & 92.68 & 99.19 & 99.23 \\
\hline Instance-based Learning & 96.94 & 97.73 & 97.25 & 97.81 & 81.50 & 94.02 & 99.22 & 99.39 \\
\hline Multilayer Perceptron & 97.57 & 98.31 & 97.69 & 98.10 & 76.56 & 93.12 & 99.45 & 99.45 \\
\hline SVM (linear kernel) & 97.63 & 97.60 & 97.67 & 97.49 & 67.79 & 88.95 & 99.28 & 99.15 \\
\hline
\end{tabular}

\subsubsection{Classifying Distances with the Reduced Feature Set}

In Section 5.1.2, we have presented that a ceiling- or a pair of wall-mounted modules equipped with a single PIR $_{1}$ sensor works well for classifying distance intervals, i.e., about $95 \%$ accuracy. We have also demonstrated the algorithms appropriate for walking distance classification, including Bayes net, instance-based learning, multilayer perceptron and support vector machine with quadratic and cubic kernels. Accordingly, we perform classification analysis based on the machine learning algorithms and 
the reduced feature set. Table 10 summarizes the experimental result with respect to the modules and features. Overall, we can know that instance-based learning shows the best performance, in particular, when using all three modules and both amplitude and time to peaks as features.

Table 10. Comparison of the recognition accuracy (\%) of classifying walking distance intervals based on the reduced feature set with respect to the modules and features (amplitude and time to peaks).

\begin{tabular}{|c|c|c|c|c|c|c|}
\hline \multirow{7}{*}{ Classifier } & \multicolumn{6}{|c|}{ Recognition Accuracy (\%) } \\
\hline & \multirow{2}{*}{\multicolumn{2}{|c|}{$\begin{array}{c}\text { Module }_{3} \\
\quad \square \\
\times \quad \times\end{array}$}} & \multicolumn{2}{|c|}{$\underset{\times}{\text { Module }_{1,2}}$} & \multicolumn{2}{|c|}{$\begin{array}{c}\text { Module }_{1,2,3} \\
\square\end{array}$} \\
\hline & & & \multicolumn{2}{|c|}{$\begin{array}{l}\square \quad \square \\
\text { DIP }\end{array}$} & \multicolumn{2}{|c|}{$\square \quad \square$} \\
\hline & \multicolumn{2}{|c|}{ 会 } & \multirow{3}{*}{\multicolumn{2}{|c|}{$\begin{array}{l}\mathrm{PIR}_{1} \\
\bigcirc \times \\
\times \times\end{array}$}} & \multirow{3}{*}{\multicolumn{2}{|c|}{$\begin{array}{l}\mathrm{PIR}_{1} \\
\bigcirc \times \\
\times \times\end{array}$}} \\
\hline & \multirow{2}{*}{\multicolumn{2}{|c|}{$\underset{x \times}{O x}$}} & & & & \\
\hline & & & & & & \\
\hline & Amplitude & $\begin{array}{l}\text { Amplitude } \\
\text { and time }\end{array}$ & Amplitude & $\begin{array}{l}\text { Amplitude } \\
\text { and time }\end{array}$ & Amplitude & $\begin{array}{l}\text { Amplitude } \\
\text { and time }\end{array}$ \\
\hline Bayes Net & 92.91 & 90.90 & 69.17 & 75.13 & 93.11 & 95.77 \\
\hline Instance-based Learning & 93.13 & 90.40 & 74.96 & 90.40 & 89.97 & 98.05 \\
\hline Multilayer Perceptron & 86.13 & 84.13 & 68.91 & 81.04 & 89.85 & 94.78 \\
\hline SVM (quadratic kernel) & 69.21 & 74.84 & 68.10 & 84.90 & 89.30 & 96.84 \\
\hline SVM (cubic kernel) & 69.59 & 75.39 & 69.65 & 87.63 & 90.32 & 97.60 \\
\hline
\end{tabular}

Table 11. Comparison of the recognition accuracy (\%) of classifying walking speed levels based on the reduced feature set with respect to the modules and features (amplitude and time to peaks and passage duration).

\begin{tabular}{|c|c|c|c|c|c|c|}
\hline \multirow{5}{*}{ Classifier } & \multicolumn{6}{|c|}{ Recognition Accuracy (\%) } \\
\hline & \multicolumn{2}{|c|}{$\begin{array}{c}\text { Module }_{3} \\
\square\end{array}$} & \multicolumn{2}{|c|}{$\begin{array}{c}\text { Module }_{1,2} \\
\times\end{array}$} & \multicolumn{2}{|c|}{$\begin{array}{c}\text { Module }_{1,2,3} \\
\square\end{array}$} \\
\hline & $\times$ & $x$ & $\square$ & $\square$ & $\square$ & $\square$ \\
\hline & $\begin{array}{l}\mathrm{PIR}_{1} \\
\bigcirc \times \\
\times \times\end{array}$ & $\begin{array}{c}\mathrm{PIR}_{1,2,3,4} \\
\bigcirc \\
\bigcirc \bigcirc\end{array}$ & $\begin{array}{l}\mathrm{PIR}_{1} \\
\mathrm{O} \times \\
\times \times\end{array}$ & $\begin{array}{c}\mathrm{PIR}_{1,2,3,4} \\
\bigcirc \\
\bigcirc\end{array}$ & $\begin{array}{l}\mathrm{PIR}_{1} \\
\mathrm{O} \\
\times \times\end{array}$ & $\begin{array}{c}\mathrm{PIR}_{1,2,3,4} \\
\bigcirc \\
\bigcirc\end{array}$ \\
\hline & $\begin{array}{c}\text { Amplitude, } \\
\text { time, and } \\
\text { passage duration }\end{array}$ & $\begin{array}{c}\text { Amplitude, } \\
\text { time, and } \\
\text { passage duration }\end{array}$ & $\begin{array}{c}\text { Amplitude, } \\
\text { time, and } \\
\text { passage duration }\end{array}$ & $\begin{array}{c}\text { Amplitude, } \\
\text { time, and } \\
\text { passage duration }\end{array}$ & $\begin{array}{c}\text { Amplitude, } \\
\text { time, and } \\
\text { passage duration }\end{array}$ & $\begin{array}{c}\text { Amplitude, } \\
\text { time, and } \\
\text { passage duration }\end{array}$ \\
\hline Instance-based Learning & 84.49 & 90.54 & 88.32 & 89.75 & 89.02 & 89.34 \\
\hline SVM (quadratic kernel) & 74.50 & 86.13 & 85.11 & 94.11 & 86.29 & 94.80 \\
\hline SVM (cubic kernel) & 78.34 & 90.58 & 87.07 & 94.29 & 89.62 & 94.94 \\
\hline
\end{tabular}

\subsubsection{Classifying Speeds with the Reduced Feature Set}

In Section 5.1.3, we have presented that a ceiling- or a pair of wall-mounted modules equipped with a single $\mathrm{PIR}_{1}$ sensor works well for classifying speed levels, i.e., about $90 \%$ accuracy. We have also demonstrated the algorithms appropriate for walking speed classification, including instance-based learning and support vector machine with quadratic and cubic kernels. Accordingly, we perform a classification analysis based on the machine learning algorithms and the reduced feature set. In this study, we have also employed passage duration as a feature, because it might depend highly on the subject's walking speed. Table 11 summarizes the experimental result with respect to the modules (i.e., 
a ceiling-mounted module $v s$. a pair of wall-mounted modules) and PIR sensors (i.e., a single PIR sensor $v s$. all four PIR sensors). Overall, support vector machine with cubic kernel shows the best performance, in particular, when involving a pair of wall-mounted modules and all four PIR sensors, though there is no big difference between the accuracy with and without the ceiling-mounted module (i.e., Module $_{3}$ ).

\subsubsection{Identifying Subjects with the Reduced Feature Set}

In Section 5.1.4, we have presented that a pair of wall-mounted modules facing each other, each of which has a single PIR sensor, could achieve about an $87 \%$ recognition accuracy for identifying eight subjects. We have also presented that all three modules, each of which has a single PIR sensor, could achieve about a $92 \%$ recognition accuracy. We have also demonstrated that the recognition accuracy could be increased (until 95\%) as the number of PIR sensors involved in the classification is increased. In addition, we have presented the algorithms appropriate for walking subject identification, including instance-based learning and support vector machine with quadratic and cubic kernels. Accordingly, we perform classification analysis based on the machine learning algorithms and the reduced feature sets. Table 12 summarizes the experimental result with respect to the modules and PIR sensors. Overall, we can know that support vector machine with quadratic and cubic kernels shows the best performance (about 96\%, even better than the result with the raw data set), in particular, when using all PIR sensors equipped in all modules. It should be also noted that the result without a ceiling-mounted PIR-based module (i.e., Module $_{3}$ ) shows more than a $91 \%$ recognition accuracy in identifying walking subjects.

Table 12. Comparison of the recognition accuracy (\%) of identifying walking subjects based on the reduced feature set with respect to the modules and PIR sensors.

\begin{tabular}{|c|c|c|c|c|c|c|}
\hline \multirow{5}{*}{ Classifier } & \multicolumn{6}{|c|}{ Recognition Accuracy (\%) } \\
\hline & \multirow{2}{*}{\multicolumn{3}{|c|}{$\begin{array}{c}\text { Module }_{1,2} \\
\times \\
\square \quad \square\end{array}$}} & \multirow{2}{*}{\multicolumn{3}{|c|}{$\begin{array}{c}\text { Module }_{1,2,3} \\
\square\end{array}$}} \\
\hline & & & & & & \\
\hline & $\begin{array}{l}\mathrm{PIR}_{1} \\
\bigcirc \times \\
\times \times\end{array}$ & $\begin{array}{l}\mathrm{PIR}_{1,3} \\
\bigcirc \times \\
\times \bigcirc\end{array}$ & $\begin{array}{c}\mathrm{PIR}_{1,2,3,4} \\
\bigcirc \bigcirc \\
\bigcirc \bigcirc\end{array}$ & $\begin{array}{l}\mathrm{PIR}_{1} \\
\bigcirc \times \\
\times \times\end{array}$ & $\begin{array}{l}\mathrm{PIR}_{1,3} \\
\bigcirc \times \\
\times \bigcirc\end{array}$ & $\begin{array}{c}\mathrm{PIR}_{1,2,3,4} \\
\bigcirc \bigcirc \\
\bigcirc \bigcirc\end{array}$ \\
\hline & $\begin{array}{c}\text { Amplitude, } \\
\text { time, and } \\
\text { passage duration }\end{array}$ & $\begin{array}{c}\text { Amplitude, } \\
\text { time, and } \\
\text { passage duration }\end{array}$ & $\begin{array}{c}\text { Amplitude, } \\
\text { time, and } \\
\text { passage duration }\end{array}$ & $\begin{array}{c}\text { Amplitude, } \\
\text { time, and } \\
\text { passage duration }\end{array}$ & $\begin{array}{c}\text { Amplitude, } \\
\text { time, and } \\
\text { passage duration }\end{array}$ & $\begin{array}{c}\text { Amplitude, } \\
\text { time, and } \\
\text { passage duration }\end{array}$ \\
\hline Instance-based Learning & 75.06 & 79.36 & 83.11 & 76.95 & 79.22 & 83.68 \\
\hline SVM (quadratic kernel) & 59.09 & 79.86 & 91.86 & 72.10 & 88.88 & 96.33 \\
\hline SVM (cubic kernel) & 66.08 & 83.24 & 92.31 & 77.41 & 89.47 & 96.56 \\
\hline
\end{tabular}

\section{Discussion}

From the experimental results, we can know that a pair of PIR-based modules mounted on opposite walls facing each other could classify the direction of movement, the distance of the body from the PIR sensors and the speed level of movement during two-way, back-and-forth walking and even identify the walking subjects. A ceiling-mounted PIR-based module could also classify the direction, distance and speed of walking subjects, but does not perform subject identification well. Accordingly, we can imagine extensions to this study adapted to building a smart environment, where a set of PIR sensors are attached on opposite walls facing each other at the entrance to the room and multiple PIR-based modules are 
distributed in a square grid across the ceiling in the room. In the smart environment, the wall-mounted PIR sensors at the entrance could identify the user entering the room, and the ceiling-mounted PIR-based modules could detect the user's movement, including direction, distance and speed, robustly tracking the user and, thus, helping the system build a rich model of the user's context.

The experimental results using only a single PIR sensor (i.e., $\mathrm{PIR}_{1}$ ) embedded in each module have shown good performance in classifying directions, distances and speeds, and this is probably because the walking samples we have used in our experiments are collected from two-way, back-and-forth walking, and $\mathrm{PIR}_{1}$ (and thus, its sensing elements) that each of the PIR-based modules is equipped with, is well aligned with the motion plane, i.e., the walking directions. However, we also found that the more PIR sensors, i.e., a pair of PIR sensors orthogonally aligned ( $\mathrm{PIR}_{1}$ and $\mathrm{PIR}_{3}$ ) or two pairs of PIR sensors orthogonally aligned ( $\mathrm{PIR}_{1}$ and $\mathrm{PIR}_{3}, \mathrm{PIR}_{2}$ and $\mathrm{PIR}_{4}$ ), could improve the recognition accuracy, in particular, in the experiment for identifying walking subjects. In addition, for other public spaces at home or work, where occupants perform multi-directional movements, ceiling-mounted PIR-based modules rather than wall-mounted PIR-based modules are appropriate for human movement detection, and of course, the more the number of PIR sensors that are involved in the modules, the better the performance becomes.

In this study, we have performed an investigation into the recognition accuracy with respect to various machine learning algorithms, including Bayes net, decision tree (C4.5), decision table, instance-based learning ( $k$-nearest neighbor algorithm), multilayer perceptron, naive Bayes and support vector machine with linear, quadratic and cubic kernels. Among them, Bayes net works well for classifying walking directions and distance levels. Multilayer perceptron also works well for classifying walking speeds, as well as walking directions and distance intervals. However, instance-based learning ( $k$-nearest neighbor algorithm) and supper vector machine, in particular, with quadratic and cubic kernels outperform all other algorithms in most analyses. Between them, instance-based learning might be a better solution, because support vector machine with quadratic or cubic kernels usually requires a greater computation load for the training data set, though instance-based learning probably requires larger memory resources to retain a set of classified (i.e., labeled) examples for performing supervised learning. Nevertheless, for the study for identifying subjects, in particular, using the reduced feature set, support vector machine with quadratic or cubic kernels may be the only solution for good performance (see Table 12).

As presented in Section 4.3, the computation cost for human movement detection and identification using the devices we have implemented will vary according to the number of modules (Module ${ }_{1}$,

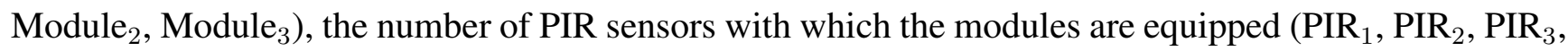
$\mathrm{PIR}_{4}$ ) and the features (raw data set or reduced feature set composed of amplitude and time to peaks, and passage duration) involved in the classification analysis. In the cases of a pair of wall-mounted modules facing each other, we could achieve almost or more than $85 \%$ recognition accuracy for classifying walking directions, distances and speeds based on the reduced feature set collected from a single PIR 1 sensor with which each module was equipped. In the study, we have used 14 features (= 2 PIR-based modules $\times 1$ PIR sensor/PIR-based module $\times 7$ features/PIR sensor). In the case of a ceiling-mounted module, we could achieve almost or more than an $84 \%$ recognition accuracy for classifying walking

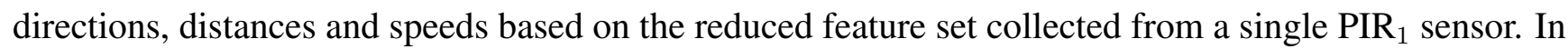
the study, we have used only seven features (= 1 PIR-based module $\times 1$ PIR sensor/PIR-based module $\times$ 
7 features / PIR sensor). However, for identifying subjects with more than a 90\% recognition accuracy, we need to employ all four PIR sensors with which each wall-mounted module is equipped, as presented in Table 12.

Our system shows a similar performance ( $>99 \%)$ to the previous PIR sensor-based systems in classifying walking directions and distance intervals. For identifying walking subjects, our system shows better performance $(96.56 \%)$ than the previous PIR sensor-based identification methods (e.g., $91 \%$ in [18], 84\% in [21]) except Hao's work (100\%) in [20]. However, we cannot claim that our PIR sensor-based identification system will perform better (or even worse) than other PIR-based systems, because the number of subjects identified are different (e.g., eight subjects for our study $v s$. five subjects for Hao's work in [20]), and the data set for identification might be collected in different walking conditions. As a consequence, although there would still be a question of which method is better in terms of recognition accuracy, we can conclude with confidence that the analog output signals of PIR sensors installed on the ceiling and walls can be used to reliably classify walking direction, the distance of the human body from the sensor and the speed level and to identify walking subjects in indoor environments.

\section{Conclusion}

We have presented a human movement detecting system based on pyroelectric infrared (PIR) sensors and machine learning technologies for classifying the direction of movement, the distance of the body from the PIR sensors, the speed of movement during two-way, back-and-forth walking and identifying the walking subject. To collect PIR sensor signals, we have built a PIR-based module consisting of two pairs of PIR sensors orthogonally aligned, op-amp circuits, a data logger and a rechargeable battery. We have placed three PIR-based modules: one module mounted on the ceiling and two modules mounted on opposite walls facing each other in a hallway. Using the PIR-based modules, we have collected PIR sensor signals when eight different experimental participants were walking through the monitoring field in three different conditions: direction, walking in two different directions (i.e., forward and backward); distance, walking at three different distances from PIR modules (i.e., close to PIR Module 1 , middle, close to PIR Module ${ }_{2}$ ); speed, walking at three different speed levels (i.e., slow, moderate, fast). Based on the data set collected from the PIR-based modules, we have performed classification analysis for detecting walking direction, distance, speed and the subject using two types of feature sets: a raw data set and a reduced feature set composed of amplitude and time to peaks and passage duration for each PIR sensor signal, and various machine learning algorithms, including instance-based learning and support vector machine. Our results show that it is feasible to detect the direction and speed of movement and the distance of the body from the PIR sensors; and identifying subjects with more than a $92 \%$ recognition accuracy using the raw data set collected from a single PIR sensor of each of the three PIR-based modules. We could also achieve more than a $94 \%$ accuracy in classifying the direction, distance and speed and identifying subjects using the reduced feature set from two pairs of PIR sensors of each of the three PIR-based modules. 


\section{Acknowledgments}

This work was supported by the IT R\&D program of MSIP (Ministry of Science, ICT, and Future Planning)/KEIT (Korea Evaluation Institute of Industrial Technology) (10044811, the development of radiosonde and automatic upper air sounding system for monitoring of hazardous weather and improvement of weather forecasting).

\section{Author Contributions}

The work presented in this paper is a collaborative development by both authors. Yun defined the research theme, designed methods and experiments, developed data collection modules and performed data analysis and classification analysis. Lee performed data collection and gave technical support and conceptual advice. Yun wrote the paper, and Lee reviewed and edited the manuscript. Both of them read and approved the manuscript.

\section{Conflicts of Interest}

The authors declare no conflict of interest.

\section{References}

1. Han, Z.; Gao, R.X.; Fan, Z. Occupancy and indoor environment quality sensing for smart buildings. In Proceedings of the 5th European DSP Education and Research Conference (EDERC'12), Graz, Austria, 13-16 May 2012; pp. 882-887.

2. Tsai, C.-H.; Bai, Y.-W.; Chu, C.-A.; Chung, C.-Y.; Lin, M.-B. PIR-sensor-based lighting device with ultra-low standby power consumption. IEEE Trans. Consum. Electron. 2011, 57, 1157-1164.

3. Tsai, C.-H.; Bai, Y.-W.; Cheng, L.-C.; Lin, K.-S.; Jhang, R.J.R.; Lin, M.-B. Reducing the standby power consumption of a pc monitor. In Proceedings of the 1st Global Conference on Consumer Electronics, Tokyo, Japan, 2-5 October 2012; pp. 520-524.

4. Erickson, V.L.; Achleitner, S.; Cerpa, A.E. POEM: Power-efficient occupancy-based energy management system. In Proceedings of the 12th International Conference on Information Processing in Sensor Networks, Philadelphia, PA, USA, 8-11 April 2013; pp. 203-216.

5. Gopinathan, U.; Brady, D.J.; Pitsianis, N.P. Coded apertures for efficient pyroelectric motion tracking. Opt. Express 2003, 11, 2142-2152.

6. Shankar, M.; Burchett, J.B.; Hao, Q.; Guenther, B.D.; Brady, D.J. Human-tracking systems using pyroelectric infrared detectors. Opt. Eng. 2006, 45, 106401.

7. Hao, Q.; Brady, D.J.; Guenther, B.D.; Burchett, J.B.; Shankar, M.; Feller, S. Human tracking with wireless distributed pyroelectric sensors. IEEE Sens. J. 2006, 6, 1683-1696.

8. Luo, X.; Shen, B.; Guo, X.; Luo, G.; Wang, G. Human tracking using ceiling pyroelectric infrared sensors. In Proceedings of the IEEE International Conference on Control and Automation, Christchurch, New Zealand, 9-11 December 2009; pp. 1716-1721. 
9. Hashimoto, K.; Morinaka, K.; Yoshiike, N.; Kawaguchi, C.; Matsueda, S. People count system using multi-sensing application. In Proceedings of the International Conference on Solid State Sensors and Actuators, Chicago, IL, USA, 16-19 June 1997; Volume 2, pp. 1291-1294.

10. Wahl, F.; Milenkovic, M.; Amft, O. A green autonomous self-sustaining sensor node for counting people in office environments. In Proceedings of the 5th European DSP Education and Research Conference, Graz, Austria, 13-16 May 2012; pp. 203-207.

11. Wahl, F.; Milenkovic, M.; Amft, O. A distributed PIR-based approach for estimating people count in office environments. In Proceedings of the IEEE 15th International Conference on Computational Science and Engineering, Paphos, Cyprus, 5-7 December 2012; pp. 640-647.

12. Lee, W. Method and Apparatus for Detecting Direction and Speed Using PIR Sensor. U.S. Patent 5,291,020, 1 March 1994.

13. Zappi, P.; Farella, E.; Benini, L. Enhancing the spatial resolution of presence detection in a PIR based wireless surveillance network. In Proceedings of the IEEE Conference on Advanced Video and Signal Based Surveillance, London, UK, 5-7 September 2007; pp. 295-300.

14. Zappi, P.; Farella, E.; Benini, L. Pyroelectric infrared sensors based distance estimation. In Proceedings of the 7th IEEE Sensors Conference (IEEE Sensors'08), Lecce, Italy, 28-29 October 2008; pp. 716-719.

15. Zappi, P.; Farella, E.; Benini, L. Tracking motion direction and distance with pyroelectric IR sensors. IEEE Sens. J. 2010, 10, 1486-1494.

16. Yun, J.; Song, M.-H. Detecting Direction of Movement using Pyroelectric Infrared Sensors. IEEE Sens. J. 2014, 14, 1482-1489.

17. Fang, J.-S.; Hao, Q.; Brady, D.J.; Shankar, M.; Guenther, B.D.; Pitsianis, N.P.; Hsu, K.Y. Path-dependent human identification using a pyroelectric infrared sensor and Fresnel lens arrays. Opt. Express 2006, 14, 609-624.

18. Fang, J.-S.; Hao, Q.; Brady, D.J.; Guenther, B.D.; Hsu, K.Y. Real-time human identification using a pyroelectric infrared detector array and hidden Markov models. Opt. Express 2006, 14, 6643-6658.

19. Fang, J.-S.; Hao, Q.; Brady, D.J.; Guenther, B.D.; Hsu, K.Y. A pyroelectric infrared biometric system for real-time walker recognition by use of a maximum likelihood principal components estimation (MLPCE) method. Opt. Express 2007, 15, 3271-3284.

20. Hao, Q.; Hu, F.; Xiao, Y. Multiple human tracking and identification with wireless distributed pyroelectric sensor systems. IEEE Sens. J. 2009, 3, 428-439.

21. Tao, S.; Kudo, M.; Nonaka, H.; Toyama, J. Person authentication and activities analysis in an office environment using a sensor network. In Constructing Ambient Intelligence; Springer Berlin Heidelberg: Berlin, Germany, 2012; pp. 119-127.

22. Sun, Q.; Hu, F.; Hao, Q. Mobile target scenario recognition via low-cost pyroelectric sensing system: Toward a context-enhanced accurate identification. IEEE Trans. Syst. Man Cybern. Syst. 2014, 44, 375-384.

23. Stauffer, C.; Grimson, W.E.L. Learning patterns of activity using real-time tracking. IEEE Trans. Pattern Anal. Mach. Intell. 2000, 22, 747-757.

24. Whatmore, R.W. Pyroelectric devices and materials. Rep. Prog. Phys. 1986, 49, 1335-1386. 
25. Milde, G.; Häusler, C.; Gerlach, G.; Bahr, H.-A.; Balke, H. 3-D modeling of pyroelectric sensor arrays part ii: Modulation transfer function. IEEE Sens. J. 2008, 8, 2088-2094.

26. Pyroelectric Infrared Sensors. Available online: http://www.murata.com/products/catalog/pdf/ s21e.pdf (accessed on 4 May 2014).

27. Cirino, G.A.; Barcellos, R.; Morato, S.P.; Bereczki, A.; Neto, L.G. Design, fabrication, and characterization of Fresnel lens array with spatial filtering for passive infrared motion sensors. SPIE Proc. 2006, 6343, 634323.

28. Fuji \& Co. (Piezo Science), Gernaral Operation. Available online: http://www.fuji-piezo.com/ TechGen.htm (accessed on 4 May 2014).

29. Weka 3: Data Mining Software in Java. Available online: http://www.cs.waikato.ac.nz/ml/weka (accessed on 4 May 2014).

30. Eclipse IDE for Java Developers. Available online: http://www.eclipse.org/ (accessed on 4 May 2014).

(C) 2014 by the authors; licensee MDPI, Basel, Switzerland. This article is an open access article distributed under the terms and conditions of the Creative Commons Attribution license (http://creativecommons.org/licenses/by/3.0/). 